\title{
Increasing women's use of the IUD for family planning
}

Ricardo Vernon

Follow this and additional works at: https://knowledgecommons.popcouncil.org/departments_sbsr-rh

Part of the Demography, Population, and Ecology Commons, Family, Life Course, and Society Commons, Gender and Sexuality Commons, International Public Health Commons, Maternal and Child Health Commons, Medicine and Health Commons, and the Women's Health Commons How does access to this work benefit you? Let us know!

\section{Recommended Citation}

Vernon, Ricardo. 2008. "Increasing women's use of the IUD for family planning," FRONTIERS Program Brief no. 9. Washington, DC: Population Council. 


\section{Increasing Women's Use of the IUD for Family Planning}

T he intrauterine device (IUD) is highly effective, easy to use, and has few side effects (Hatcher et al. 1997). The device costs the provider about US $\$ 2.00$ and offers a duration of protection per unit that makes it the most cost-effective of the temporary methods if used for two years or more (Chiou et al. 2003).

The IUD's many advantages also make it a very good alternative for reducing unmet need for long-term contraception around the world. Between 5 and 17 percent of all married women of reproductive age in 53 lessdeveloped countries do not want to have more children but are not using a contraceptive method (Ashford 2003). Because the IUD can be provided in primary health centers and posts at low cost, it can complement female sterilization and vasectomy to help women achieve their fertility goals, especially in rural areas.

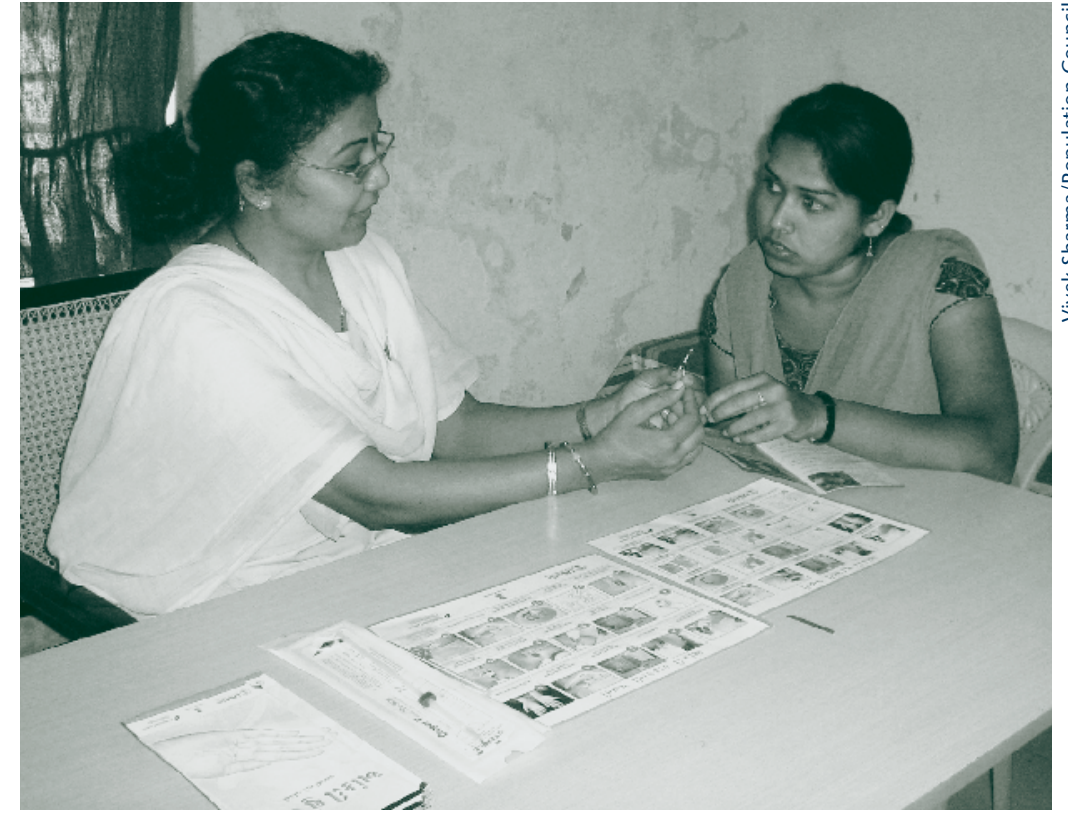

IUD counseling at a health center in Gujarat, India.

Despite these advantages, the IUD is underused. Only 5 percent of married women in developing countries other than China ${ }^{1}$ (1\% in Africa, $4 \%$ in Asia outside China, and 8\% in Latin America and the Caribbean) use the method (Salem 2006).

1In China, 84 percent of married women use a family planning method, and of those, 43 percent use IUDs (Salem 2006).
The Frontiers in Reproductive Health Program (FRONTIERS) conducted projects in Africa, Asia and the Near East, and Latin America and the Caribbean to understand why the IUD is not used more extensively and to test strategies to make it more accessible and acceptable for couples. This brief describes findings from these studies and suggests approaches for increasing the availability and use of IUD services.

The IUD is a safe, effective, cost-effective, and reversible long-term family planning method.

\section{- Improving provider knowledge and skills and conducting outreach to clients can increase use of the IUD.}

Both professional and paraprofessional providers can deliver IUD services safely. 


\section{Advantages of the IUD}

One pregnancy (or less) per 125 users in the first year of use

- Protection up to 10 years

- Immediately reversible

Can be used during lactation and menopause

No adverse reaction to medication, including antiretroviral therapy

Few side effects

Convenient for users (annual checkups compared to more frequent checkups for users of other methods, thus lower costs for revisits and resupplies)

- Can be inserted by trained providers at nearly any clinic

(Sources: Hatcher et al. 1997; Salem 2006)

Reasons for low IUD use

FRONTIERS conducted studies in Ghana (Gyapong et al. 2003; Osei et al. 2008); India (Khan et al. 2007) and Guatemala (Brambila and Taracena 2003) to assess the reasons for low IUD use. These studies found that IUD use is affected by a variety of factors having to do with the delivery of IUD services the demand for IUDs:

\section{- Lack of equipment and trained staff to provide} the services. In Guatemala, for example, only one-third of the Ministry of Health (MOH) health centers had both the basic equipment and at least one trained staff member to provide IUDs. Although most studies found that only a minority of providers had negative perceptions of the method, they seemed to lack confidence in their skills to deliver the method, suggesting the need for more extensive training to maintain competency among those who have been trained.

\section{Insufficient knowledge} among providers. In India about half of health care providers did not recognize that IUD use was contraindicated in the presence of sexually transmitted infections (STIs), and about one-fourth did not know the IUD's effective duration.

\section{Insufficient information to potential clients. In} Guatemala, analysis of information given to simulated clients showed that providers mentioned only 12 percent of the information items about IUDs that they should give clients during counseling sessions according to the family planning service delivery guidelines. In Ghana, the IUD was not mentioned in nearly one-fourth of the counseling sessions.

\section{Policy and programmatic} barriers. In many countries, only doctors and nurses are allowed to provide IUD services, even though nurse auxiliaries and other paramedical providers staff most of the health centers and posts. Also, many programs offer the IUD only in the interval period (after the six-week postpartum period) and do not offer it to postpartum and postabortion clients.

\section{"The IUD can make} you menstruate five times in a month..."

\section{"Sometimes it can} tilt to the side and may result in pregnancy..."

- Comments of focus group participants in Ghana reflect common misconceptions about the IUD (Osei et al. 2008) 
Box 2.

\section{Indicators to evaluate training results}

FRONTIERS OR projects used innovative indicators that are rarely reported but should become an integral part of reports evaluating training activities, including:

- Number of service providers beginning and ending theoretical and practical training by type of provider

- Number and proportion approving practical training (certified to provide the service)

- Number and proportion who conducted IUD insertion and removals in their service delivery units in the year after completing training

- Number of services provided during the following year

- Duration of training

- Cost of training per certified agent

- Impact of training on total number of services provided by service delivery unit

week to complete a minimum of five insertions (or as many as needed by the trainee) to achieve competency in IUD insertion procedures. Competency in removal was achieved using anatomical models. However, only 62 percent of the 183 nurse auxiliary trainees were able to complete their training, mostly because the demand for IUD insertions was not sufficient to enable them to conduct the supervised insertions. Further, a large proportion of those who completed training did not insert IUDs on return to their facilities, mainly because they did not feel confident in their skills and because they did not conduct demand-generating activities in their communities (Villanueva et al. 2001).

\section{Training in providers'} facilities. In Guatemala problems of low demand were addressed by conducting training at the service delivery units. Once trainees had identified potential IUD clients, they were trained in their facilities (68 health centers and nine health posts). During their visits, trainers used a checklist of itemized service delivery protocols to monitor the availability of materials, supplies, and forms, and to assess the trainees' mastery of counseling and clinical procedures. Follow-up visits showed that three-fourths of the nurse auxiliaries who completed the training (42 of 56) were still conducting insertions between 9 and 20 months after the end of the training. Thus, this training model seemed to better institutionalize the delivery of IUD services than training in clinics that provided a high volume of IUD services. However, the time needed for training was a problem: just over half (90 of 160) of the nurses and nurse auxiliaries who began training completed it, mainly because of job rotation or misunderstanding of the time commitment required for training (Montufar et al. 2005).

\section{Mobile training and delivery models}

Research in both Guatemala (Montufar et al. 2005) and Honduras (Villanueva et al. 1998) tested the effect of provider training in health fairs. In this model, providers at a health center informed community members that on a specific day they could receive a set of reproductive health services that were normally not available (such as the Pap test and the IUD) and invited them to attend on that day. Thus they concentrated demand for the service on one single day, allowing for training of providers. Although several teams used this model and recommended it highly, data to evaluate the model were not collected. This model is commonly used in Asia, with welldocumented results. Additional operations research might be used to assess the effectiveness of this approach in other areas. 
basic edu-

cation and

between one

and two years

of health train-

ing) and their

supervisors car-

ried out 2,030

IUD insertions

over a period of

12 months with

the subsequent

detection of only

three pregnancies

and no perforations

or other complica-

tions. Observation of

the service delivery

activities with the aid

of a checklist showed

that nurse auxiliaries per-

formed a high proportion

of the steps included in

the recommended service

delivery protocol, including pro-

viding appropriate information

and correctly implementing the

clinical steps itemized in the

protocol (Villanueva et al. 1998).

In Guatemala 45 trained nurse

auxiliaries conducted a monthly

mean of 1.2 insertions during a

four-month period, with only

one pregnancy and one perfora-

tion that was appropriately han-

dled. In Ghana 12 community

health officers (CHOs) in rural

areas conducted 33 insertions in

community compounds, com-

pared to only four insertions

in similar compounds without

trained CHOs over a 12-month

period; no complications were

reported (Montufar et al. 2005;

Osei et al. 2008).

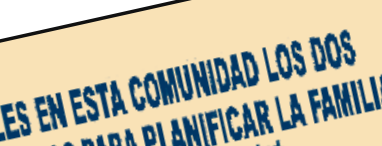

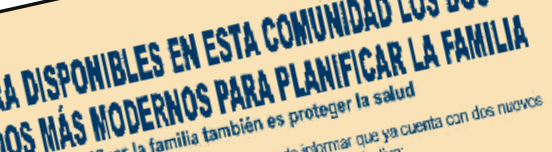

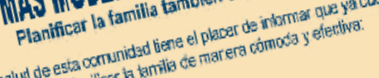

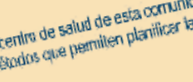

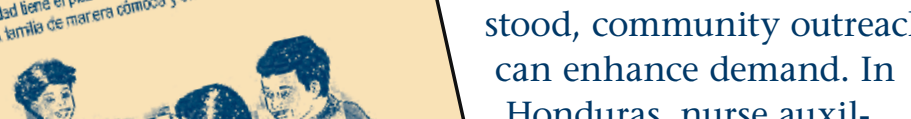

Honduras, nurse auxiliaries at six rural health centers (RHCs) gave daily 10-minute talks about new services (Pap smears, Depo-Provera, and IUDs) and asked each woman visiting the RHCs to distribute five leaflets to friends and neighbors (see Figure). Nurse auxiliaries at six control centers did not conduct outreach. New IUD users increased from 2.8

to 4.5 , new injectable users increased an average of 1.8 users per month and Pap smears increased by 1.6 samples per month. However, the only statistically significant increase was for injectable users (Mendoza and Vernon 2001).

Training alone does not guarantee use of IUD services. For example, a second study in Honduras tested the effects of an expanded training for nurse auxiliaries. A year after the training, more than half of the nurse auxiliaries who completed training had not inserted any IUDs because of low demand (Villanueva et al. 2001). This suggests that there is a need for outreach to advertise available services, as well as to counteract misperceptions about the method.
However, given the promising results, FRONTIERS worked with the $\mathrm{MOH}$, the Honduran Family Planning Association, ASHONPLAFA, and EngenderHealth on an expanded study. Forty-one health centers and posts were randomly assigned to either the experimental or control group. In the experimental group, providers were trained to conduct client and community information activities. They were given a set of informational materials including: (1) a manual for communicating information about reproductive health services, 
(2) a half-letter-sized flyer and a double-letter-sized poster highlighting the key attributes of the method, and (3) a brochure explaining the characteristics, advantages, and disadvantages of the IUD. In the experimental group, the monthly average of IUDs delivered by the health center doubled from 1.1 to 2.0, whereas in the control group it decreased from 1.7 to 0.8 , probably as a consequence of the emergency raised by two hurricanes that struck Honduras while the experiment was under way (see Table 1). The intensity with which the health centers tions strategy was correlated with a corresponding increase in the number of insertions.

These results suggest a potentially significant increase in access to IUDs on a larger scale: if the strategy were expanded to all 1,108 $\mathrm{MOH}$ health centers in Honduras, the annual number of IUD acceptors could possibly implemented the communica-

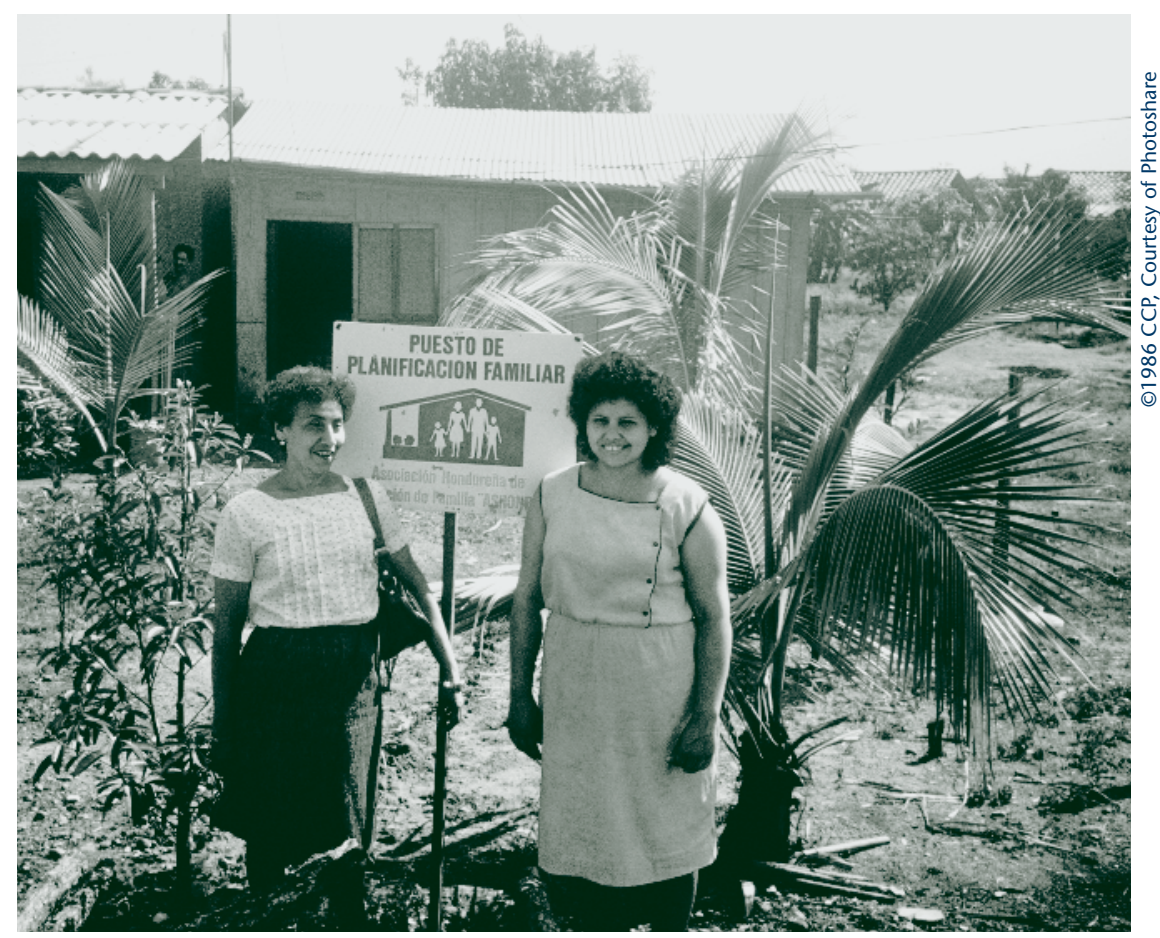

An ASHONPLAFA family planning clinic in Honduras.

increase from 11,500 to about 20,000 new IUD users. Moreover, clinic records showed that the strategy attracted women who were most in need of family planning services (Flores et al. 2007).

Table 1.

\section{Total and mean monthly number of IUDs inserted by group and time period}

\begin{tabular}{lcccc|}
\multicolumn{1}{c}{ Period } & \multicolumn{4}{c}{ Group } \\
& \multicolumn{2}{c}{ Experimental } & \multicolumn{2}{c}{ Control } \\
& Total & Mean & Total & Mean \\
& 211 & 1.1 & 310 & 1.7 \\
$\begin{array}{l}\text { January - September 2005 } \\
\text { (pre-intervention) }\end{array}$ & 294 & 2.0 & 125 & 0.7 \\
$\begin{array}{l}\text { October 2005 - May 2006 } \\
\text { (post-intervention) }\end{array}$ & 83 & 0.88 & -185 & -0.94 \\
$\begin{array}{l}\text { Before/after difference } \\
\text { (p<.05) }\end{array}$ & & & & \\
\hline
\end{tabular}

Source: Flores et al. 2007.
Other studies also highlight the importance of conducting community information activities to increase demand for littleknown, long-term methods:

- In Gujarat, India, strengthening the technical skills of 76 paraprofessionals and distributing leaflets and posters about the IUD increased the number of insertions from 204 to 262 per month at each participating facility in urban settings, and from 40 to 107 per month at each facility in rural areas (Khan et al. 2007).

- In Ghana, community health officers and community health volunteers were given IUD updates and refresher training

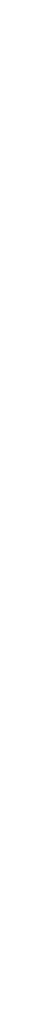

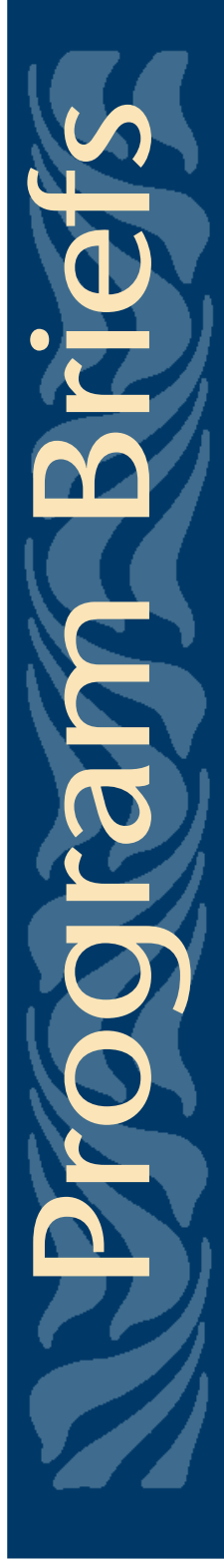


in counseling skills and were encouraged to increase IUD awareness among community members through communitybased structures and processes. Knowledge of long-term methods increased as did the proportion of respondents who reported obtaining information about long-term methods from community health officers and community volunteers (Osei et al. 2008).

\section{Providing IUDs to women in the postpartum and postabortion periods}

Insertion of the IUD addresses

a need for contraception among postpartum and postabortion women. The World Health Organization (WHO) and other international organizations recommend that women wait at least two to three years between births. Studies supported by USAID suggest that a three- to five-year interval could help reduce maternal and infant risks even further (WHO 2004; WHO 2006). Because the IUD is a long-term method that does not interfere with lactation and can be inserted at any time except between the third and 30th day after delivery (to minimize the risk of expulsion or uterine perforation), it is one of the best choices for women in the postpartum period.

OR studies have shown that it is it feasible and acceptable to provide IUD service at this time and further, that contraceptive prevalence six months after delivery is higher among those women who delivered in hospitals offering postpartum IUD services (Foreit et al. 1993). Studies in Guatemala and Honduras showed that a majority of postpartum women ( $71 \%$ and $85 \%$, respectively) wanted to use a family planning method, but only a minority (12\% and $10 \%)$ received one (Brambila, Figueroa, and Taracena 2002; Medina et al. 2001).

These results served as the basis for an intervention in five hospitals in Honduras. Clinics in participating hospitals received outreach and educational materials, equipment and supplies, and staff in OB-gyn wards attended a 30-hour counseling and service delivery workshop and a five- day contraceptive methodology workshop. Sixty-five providers were certified to provide postpartum IUD services, mostly nurses and nurse auxiliaries, who attended the majority of the deliveries at the five hospitals. In addition, the technical staff and the project coordinator held quarterly meetings in each hospital to discuss the data collected in small quarterly surveys of users as well as service statistics. The project had a significant effect on the proportion of clients who were offered, and received, contraceptive information and services, including the IUD (see Table 2).

By the endline survey, less than one year after the beginning of project activities, 33 percent of all women had received a method, and half of these had requested an IUD (Medina et al. 2001).

Client medical histories have also shown that a large proportion of women who accept an

Table 2.

\section{Percentage of postpartum women who received family planning information and services, Honduras}

\begin{tabular}{lcc|} 
& Pre-intervention & Post-intervention \\
Received FP information & 43 & 87 \\
Offered FP method & 42 & 82 \\
Received method & 10 & 33 \\
$\begin{array}{l}\text { Wanted method but did } \\
\text { not receive }\end{array}$ & 41 & 7
\end{tabular}

Source: Medina et al. 2001. 
IUD in outpatient clinics are breastfeeding women in the first six months post-delivery (see, for example, Flores et al. 2007) and that the IUD is very well accepted in postnatal care visits at six weeks (Vernon et al. 1993). Thus, outpatient clinics should emphasize presenting the IUD as an option during this time.

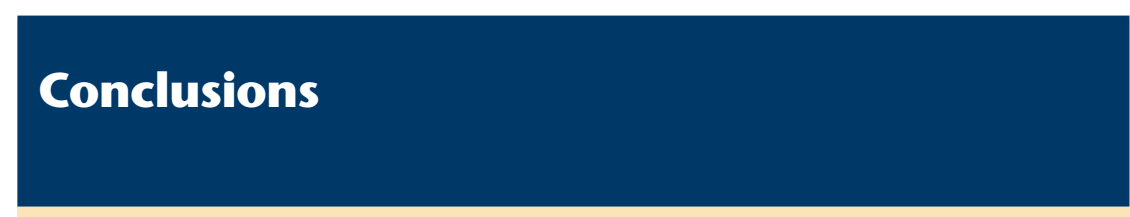

The IUD is a safe, effective, and cost-effective method, but it is also one of the least well-known methods among both providers and potential clients. To increase use of the IUD, programs need to train providers appropriately both in counseling and contraceptive technology, and conduct outreach activities to increase knowledge of the method among potential users.

Programs in low-demand settings should consider training providers onsite and allow appropriately trained paraprofessional staff to provide IUD services.

Programs should also look to introducing the IUD as a contraceptive option before patients are discharged from the hospital after delivery, miscarriage, or abortion. Postpartum insertion of IUDs offers such advantages as ease of insertion, appropriate facilities, convenience for the woman, and lower cost. The IUD can also help address an unmet need for contraception among women who seek outpatient services in the six-week to six-month postpartum period. These women should be offered IUDs as part of a full range of contraceptive methods. 


\section{Frontiers in Reproductive Health Program Brief No. 9}

Author: Ricardo Vernon

Editor: Stephanie Joyce

Design: Vincent Hughes Visualization

The assistance of the following reviewers is appreciated: Ian Askew, Laura Raney, Estela Rivero-Fuentes, Patricia Stephenson, John Townsend.

Suggested citation: Vernon, Ricardo. 2008. "Increasing Women's Use of the IUD for Family Planning," FRONTIERS Program Brief no. 9. Washington, DC: Population Council.

The photos on pages 5 and 7 were selected from Photoshare at www.photoshare.org

The Frontiers in Reproductive Health Program (FRONTIERS) applies systematic research techniques to improve delivery of family planning and reproductive health services and influence related policies. FRONTIERS is funded by the U. S. Agency for International Development (USAID) and led by the Population Council in collaboration with Family Health International.

This publication is made possible by the generous support of the American people through the United States Agency for International Development (USAID) under the terms of Cooperative Agreement No. HRN-A-00-98-00012-00. The contents are the responsibility of the FRONTIERS Program and do not necessarily reflect the views of USAID or the United States Government.

(C) 2008 The Population Council, Inc.

This publication may be reproduced in whole or in part without permission of Population Council provided full source citation is given and the reproduction is not for commercial purposes.

ISSN 1546-6612

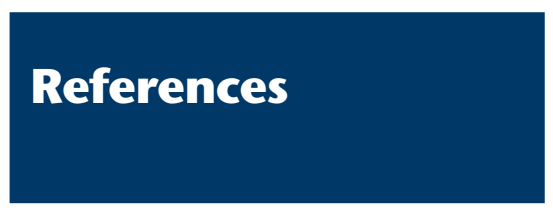

Ashford, Lori. 2003. "Unmet need for family planning: Recent trends and their implications for programs," Policy Brief. Washington DC: Population Reference Bureau, MEASURE Communication.

Brambila, Carlos and Berta Taracena. 2003. "Availability and acceptability of IUDs in Guatemala," FRONTIERS Final Report. Washington, DC: Population Council.

\section{Brambila, Carlos, Werner} Figueroa and Berta Taracena. 2002. "Situational analysis of post-obstetric event services in public hospitals in Guatemala," FRONTIERS Final Report.

Guatemala: Population Council.

Chiou, C.F. et al. 2003.

"Economic analysis of contraceptives for women," Contraception 68(1):3-10.

Family Health International (FHI). 2007. "How to Be Reasonably Sure a Client is Not Pregnant" (pregnancy checklist). Research Triangle Park, North Carolina: FHI.

Flores, Ivo et al. 2007. "Increasing use of the IUD through community and clinic based education activities in rural Honduras," FRONTIERS Final Report. Washington, DC: Population Council.
Foreit, Karen G. et al. 1993.

"Effectiveness and cost-effectiveness of postpartum IUD insertion in Lima, Peru," International Family Planning Perspectives 19(1):19-24, 33.

Gyapong, John et al. 2003. "An assessment of trends in the use of the IUD in Ghana," FRONTIERS Final Report. Washington, DC: Population Council.

Hatcher, Robert A. et al. 1997. The Essentials of Contraceptive Technology. Baltimore: The Johns Hopkins School of Public Health, Population Information Program.

Khan, M.E. et al. 2007.

"Repositioning of IUD program in Gujarat: Findings from baseline survey," FRONTIERS Research Update No. 11. New Delhi: Population Council.

Khan, M.E et al. 2007. "The Model Works! Interim findings from an OR project on repositioning of IUD in Gujarat," FRONTIERS Research Update No. 12. New Delhi: Population Council.

Melián, M., J.M. Carron, R. Vernon, and J. Garcia Nuñez. 1989. "In search of a rural family planning service delivery model in Paraguay," Final Technical Report. Asunción, Paraguay: Centro Paraguayo de Estudios de Población (CEPEP) and Population Council. 
Medina, Ruth, Ricardo Vernon, Irma Mendoza and Claudia Aguilar. 2001. "Expansion of postpartum and postabortion contraception in Honduras," FRONTIERS Final Report. Washington, DC: Population Council.

Mendoza, Irma and Ricardo Vernon. 2001. "Promoting reproductive health services in rural communities in Honduras," FRONTIERS Final Report. Washington, DC: Population Council.

Montufar, Edwin et al. 2005. "Improving access to longterm contraceptives in rural Guatemala through the Ministry of Health," FRONTIERS Final Report. Washington, DC: Population Council.

Osei, Ivy et al. 2008. Comparing the effectiveness and costs of alternative strategies for improving access to information and services for the IUD in Ghana. FRONTIERS Final Report. Washington, DC, Population Council.

Population Reference Bureau (PRB). 2002. "Family planning worldwide," 2002 Data Sheet. Washington, DC: PRB.
Ramos, Zambrano M. et al. 1985. "An experiment to improve the efficiency, effectiveness and costeffectiveness of the IUD insertion and medical back-up component of a community based distribution program in Lima, Peru," Final Report. Lima, Peru: Instituto Peruano de Paternidad Responsible (INPPARES) and Population Council.

Salem, R. 2006. "New attention to the IUD: Expanding women's contraceptive options to meet their needs," Population Reports, Series B, No. 7. Baltimore: Johns Hopkins Bloomberg School of Public Health, The INFO Project, February.

Vernon, Ricardo; Jose Ruben Lopez-Canales, Jose Arnulfo Carcamo, and Judith Galindo. 1993. "The impact of a perinatal reproductive health program in Honduras," International Family Planning Perspectives 19(3):103-109.
Villanueva, Y., L. Hernández, I. Mendoza, and R. Lundgren. 1998. "Expansion of the auxiliary nurses' role in the offering of family planning services and in the taking of Pap smears," INOPAL III Final Report. Tegucigalpa, Honduras: Population Council.

Villanueva, Y. et al. 2001. "Expansion of the role of nurse auxiliaries in the delivery of reproductive health services in Honduras," FRONTIERS Final Report. Washington, DC: Population Council.

World Health Organization (WHO). 2004. Medical Eligibility Criteria for Contraceptive Use, Third Edition. Geneva: WHO.

World Health Organization. 2006. Report of a WHO Technical Consultation on Birth Spacing. Geneva: WHO.
For more information on the IUD, see the IUD Toolkit, http://www.maqweb.org/iudtoolkit/. The Population Council was one of the members of the IUD Subcommittee of USAID's Maximizing Access and Quality Initiative that compiled the toolkit, which provides comprehensive, standardized, scientifically accurate, and evidence-based information on the IUD. The toolkit also provides guidance on best practices as well as tools to help improve access to and quality of IUD services.

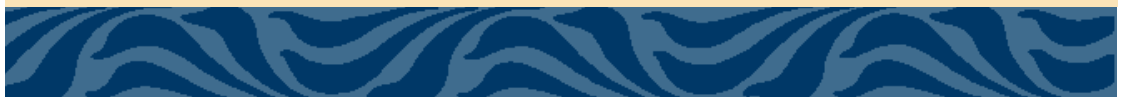


The Population Council conducts research worldwide to improve policies, programs, and products in three areas: HIV and AIDS; poverty, gender, and youth; and reproductive health.

The Frontiers in Reproductive Health Program (FRONTIERS) applies systematic research techniques to improve delivery of family planning and reproductive health services and influence related policies. FRONTIERS is funded by the U. S. Agency for International Development (USAID) and led by the Population Council in collaboration with Family Health International.

Frontiers in Reproductive Health Program (FRONTIERS) Population Council

4301 Connecticut Avenue, NW, Suite 280

Washington, DC 20008

Telephone: (202) 237-9400

Facsimile: (202) 237-8410

E-mail: publications@popcouncil.org

www.popcouncil.org/frontiers

This publication is made possible by the generous support of the American people through the United States Agency for International Development (USAID) under the terms of Cooperative Agreement No.

HRN-A-00-98-00012-00. The contents are the responsibility of the FRONTIERS Program and do not necessarily reflect the views of USAID or the United States Government.

\section{(c) 2008 The Population Council, Inc.}

This publication may be reproduced in whole or in part without permission of Population Council provided full source citation is given and the reproduction is not for commercial purposes. 